Physics, Chemistry, and Dynamics of Interplanetary Dust

ASP Conference Series, Vol. 104, 1996

Bo A. S. Gustafson and Martha S. Hanner (eds.)

\title{
SIRTF: A Unique Opportunity for Probing the Zodiacal Cloud
}

\author{
Sumita Jayaraman and Stanley F. Dermott \\ The University of Florida Department of Astronomy, 211 Space \\ Sciences Building, Gainesville, FL 32611-2055 \\ Michael Werner \\ JPL, MS 233-03, 4800 Oak Grove Dr., Pasadena, CA 91109
}

\begin{abstract}
The Space Infrared Telescope Facility (SIRTF) is planned for launch by NASA in 2001 in a heliocentric orbit at $1.01 \mathrm{AU}$. The spacecraft will drift away from the Earth slowly, reaching a distance of $0.3 \mathrm{AU}$ behind the Earth at the end of its 2.5 year mission. This implies that SIRTF will spiral through the Earth's resonant dust ring (Wright et al., 1995) and, in particular, that it will traverse the dust cloud in the ring that trails the Earth in its orbit. We have used a dynamical model of the ring (Dermott et al., 1994) followed by simulation of the SIRTF orbit to predict the variations in the zodiacal thermal emission due to the trailing dust cloud as seen by SIRTF. Because the dust ring is inclined to the ecliptic, the latitude of peak flux of the trailing cloud will have yearly oscillations about the ecliptic. The amplitude of the oscillations will increase as SIRTF approaches the cloud, reaching a maximum of $20^{\circ}$ during the mission. The magnitude of the flux variations can be as high as $4-5 \%$ or $2-3 \mathrm{MJy} / \mathrm{Sr}$. SIRTF's measurements of these effects will allow us to model the number density and thermal characteristics of asteroidal dust particles near the Earth.
\end{abstract}

\section{The SIRTF Mission}

SIRTF is a future mission planned to be launched by NASA in 2001 and will be the first infrared observatory in space to leave the confines of the Earth. The spacecraft is to be launched by a Delta rocket into a heliocentric orbit at 1.01 $\mathrm{AU}$. It will drift slowly away from the Earth at the rate of $\approx 0.1 \mathrm{AU} / \mathrm{yr}$ and trail $0.3 \mathrm{AU}$ behind the Earth at the end of the mission. Therefore, the Earth and the Moon will be negligible sources of heat with only the Sun to be blocked out using shields. A new 'warm launch' architechture has been adopted to reduce system mass and cryogenic requirements. SIRTF combines the intrinsic sensitivity of a cryogenic space telescope with the powerful imaging and spectroscopic capabilities of infrared detector arrays. It will have very high sensitivity to diffuse sources because of the ability to sum a large number of pixels after separating the discrete sources from the background radiation. Like its predecessors, the IRAS and COBE missions, SIRTF will be able to make very high signal-to-noise 


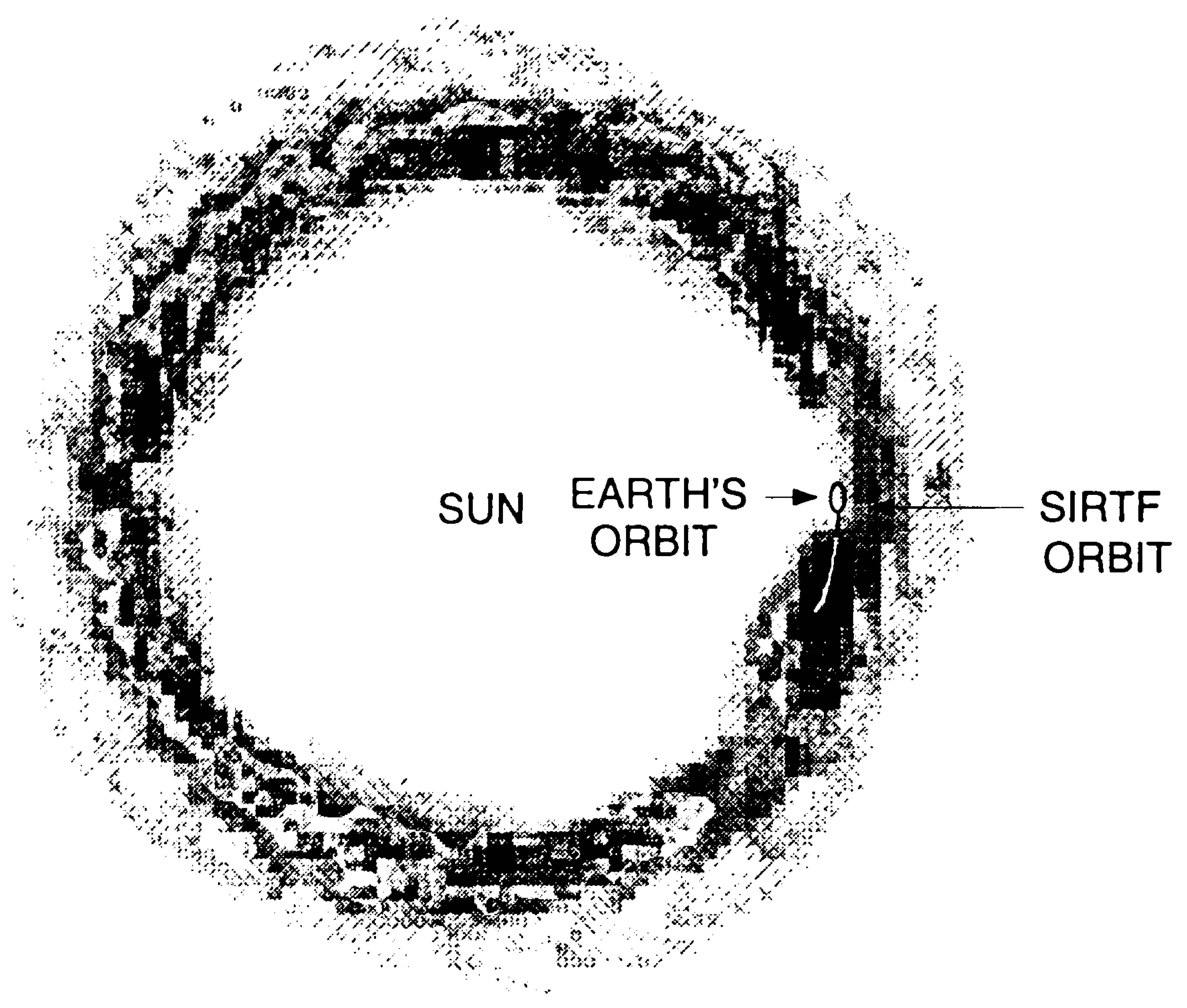

Figure 1. A model of the Earth's resonant ring in a frame rotating with the mean angular velocity of the Earth. The epicycle of the Earth's eccentric orbit as well as the orbit of SIRTF through the trailing dust cloud are also shown.

measurements of the infrared emission from interplanetary dust. The table below gives a brief overview of the mission characteristics of SIRTF. Further details on the design of SIRTF and instrumentation are given by Werner and Fanson (1995) and in a recently released Baseline Description (JPL, 1995).

\begin{tabular}{ll}
\hline \multicolumn{2}{c}{ SIRTF: Mission Summary } \\
\hline & $85 \mathrm{~cm}$ \\
Aperture & $>2.5$ years \\
Lifetime & $<6.5 \mu \mathrm{m}$ \\
Diffraction Limit & $<3$ arcsec \\
Image Size & $<0.3$ arcsec \\
Pointing Stability & Solar \\
Orbit & up to $5^{\prime} \times 5^{\prime}$, each band \\
Field of View & $3.5,4.5,6.3,8$, \\
Imaging bands & $12,30,70,160 \mu m$ \\
\hline
\end{tabular}

\section{Study of the Trailing Dust Cloud by SIRTF}

SIRTF provides a new platform to study the interplanetary dust emission. Although it will not be possible to repeat the extensive observations of DIRBE, very sensitive observations can be made far away from the Earth. The unique feature of the orbit of SIRTF is that it traverses through the trailing dust cloud embedded in the Earth's circumsolar resonance ring. The ring is formed by zo- 


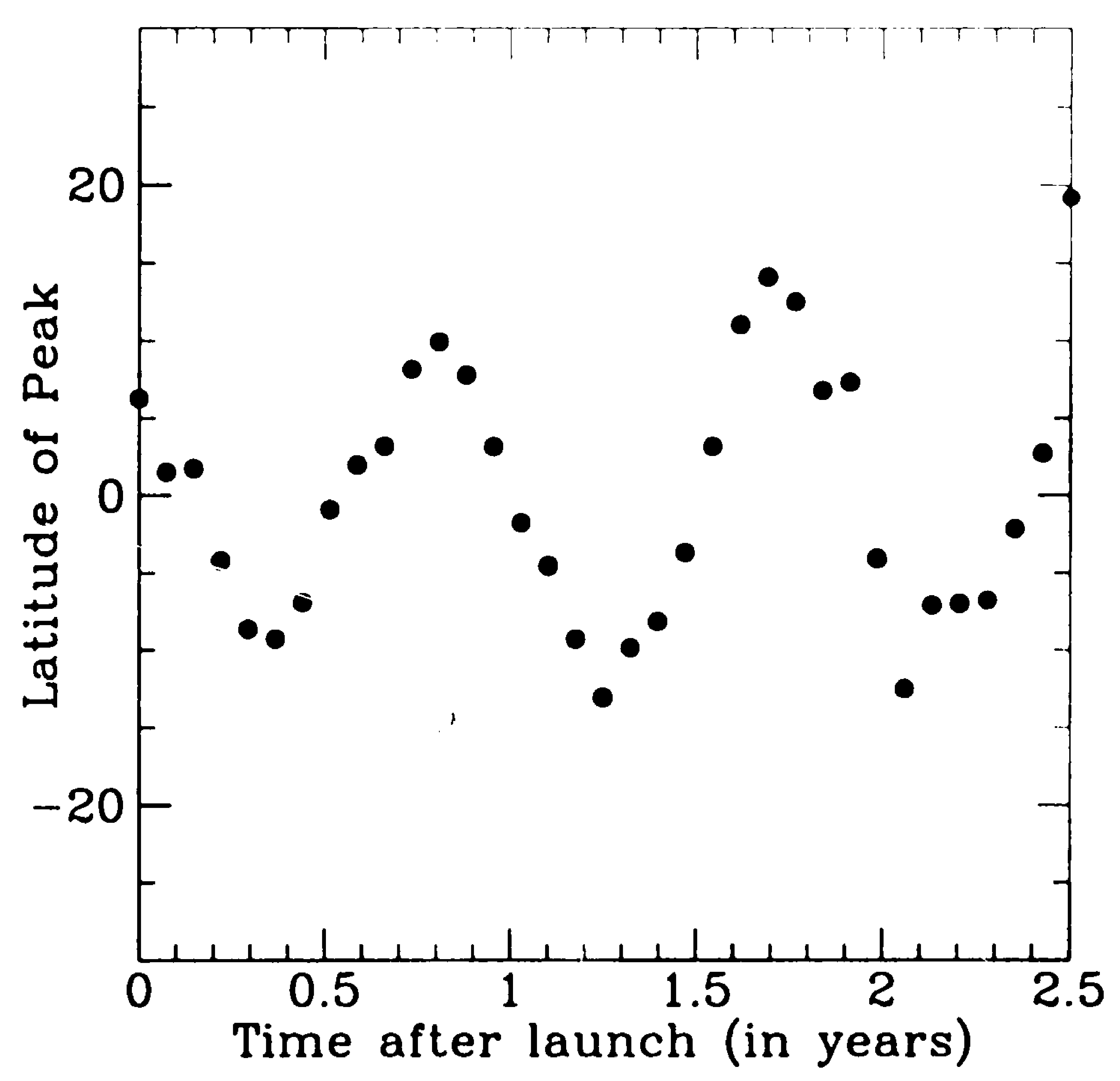

Figure 2. Predicted variation of the latitude of the peak flux of the trailing dust cloud as SIRTF approaches the cloud during its 2.5 year mission.

diacal dust particles in low eccentricity orbits (primarily asteroidal) that spiral in towards the Sun due to radiation drag forces and are trapped in the Earth's outer resonances (Dermott et al., 1994). In a frame rotating with the mean angular velocity of the Earth, the ring is a toroidal structure between 0.8 and 1.3 AU, with an enhancement in the particle number density behind the Earth which is the trailing dust cloud. The trailing dust cloud leads to a $3-4 \%$ increase in the peak flux of the zodiacal cloud in the trailing direction corresponding to 2-3 $\mathrm{MJy} / \mathrm{Sr}$ in the 25 micron waveband. A two dimensional projection of the three dimensional dynamical model of the ring for $12 \mu \mathrm{m}$ particles is shown in Figure 1 along with the Earth's $2: 1$ epicycle and the orbit of SIRTF. At the end of the mission SIRTF reaches beyond the point where the number density of particles in the trailing cloud is maximum.

The ring is inclined to the ecliptic due to the 'forced' inclination on the particles arising from planetary perturbations (Jayaraman and Dermott, 1995). Hence, the trailing dust cloud oscillates above and below the ecliptic during the course of one year. Due to the continuous change in the viewing geometry of the trailing dust cloud during the mission, there are systematic variations in the location, shape and the IR brightness of the trailing cloud. The yearly oscillation in the latitude of peak flux of the trailing cloud increases in amplitude as SIRTF approaches the cloud reaching a latitude as high as $20^{\circ}$ (Figure 2). From the numerical model of the ring shown in Figure 1, we have simulated the view of the ring as seen by SIRTF (Figure 3) at the beginning and at the end of the mission. The structure of the trailing cloud changes considerably at the end of mission and reaches higher latitudes, where the background zodiacal cloud flux decreases, maintaining the enhancement at $4-5 \%$, easily measurable by SIRTF. Comparing the predicted variations of the model to the actual observations from SIRTF we will obtain detailed information on the number density of particles across the trailing cloud. This will enable us to map the particle number density variations in near Earth region of the interplanetary dust cloud. 

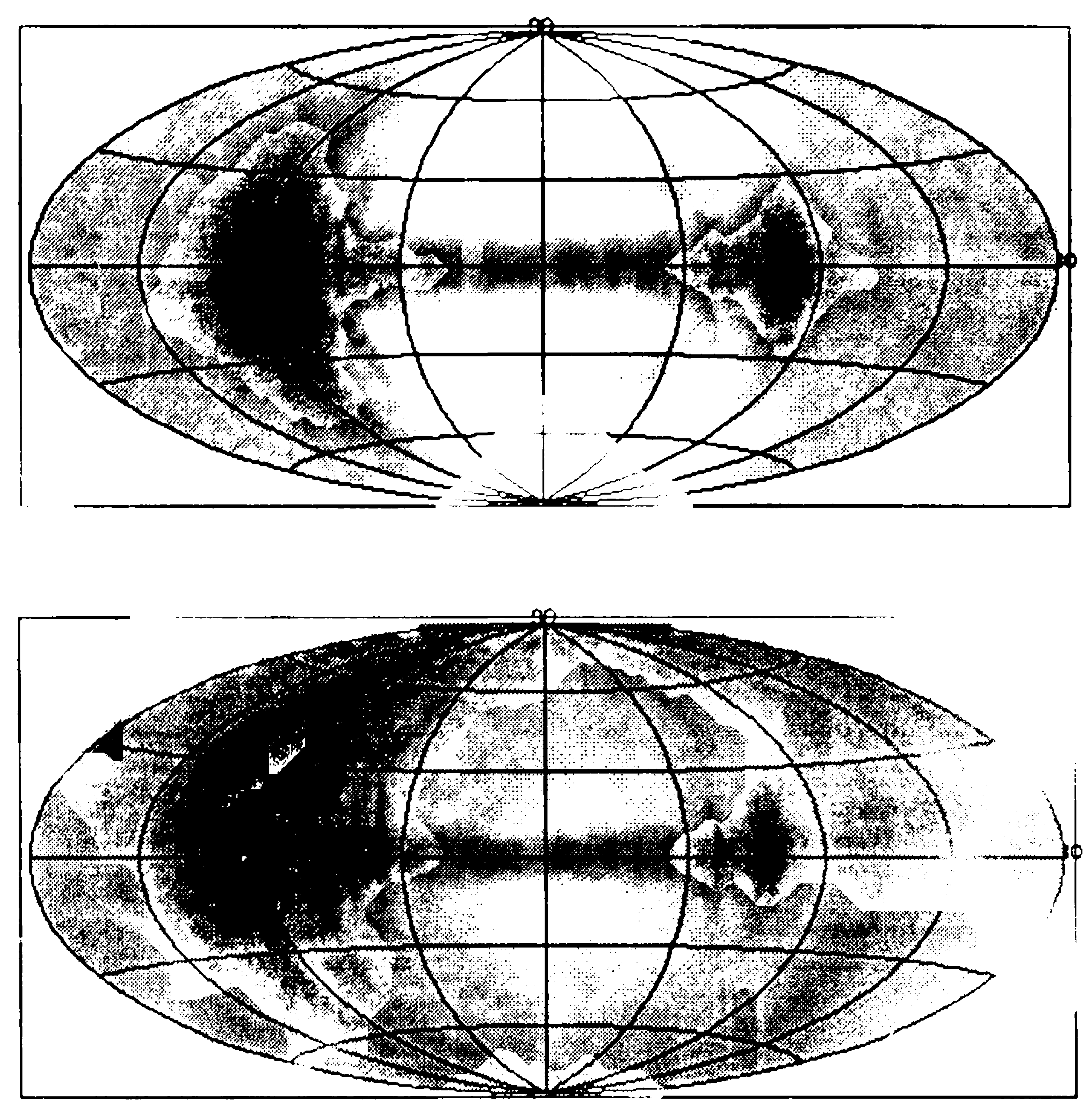

Figure 3. The view of the ring as seen by SIRTF at the beginning (top) and the end (bottom) of mission. The sun is at the center of the image and the trailing cloud is to the left.

The study of the near-Earth environment is very important in order to model the zodiacal cloud accurately because over $50 \%$ of the infrared emission comes from the dust within $0.5 \mathrm{AU}$ of the Earth. To separate the flux variations due to the resonance ring from the variations intrinsic to the zodiacal emission, good spatial and temporal coverage of the IR brightness is required. The SIRTF mission in combination with detailed dynamical models can play a key role in probing the dust distribution in the trailing direction of the Earth.

Acknowledgements Portions of this work were carried out at the Jet Propulsion Laboratory, Caltech, under contract with NASA.

\section{References}

Dermott, S.F. et al. 1994, Nature 369, 719-723

Jayaraman S. \& S.F. Dermott 1995, to be published in the Proceedings of the workshop on Unveiling the Cosmic Infrared Background.

JPL Document D-12375, Rev A, "SIRTF Baseline Observatory Design for a Delta Launch", 1995.

Werner, M.W. \& J.L. Fanson 1995. Proc. S.P.I.E., 2475, 428-437 and following papers.

Wright, E.L. et al. 1995, to be published in the Proceedings of the workshop on Unveiling the Cosmic Infrared Background. 